\title{
Food Intake, Diet Quality and Behavioral Problems in Children: Results from the GINI-plus/LISA-plus Studies
}

\author{
Gabriele Kohlboeck $^{\mathrm{a}}$ Stefanie Sausenthaler ${ }^{\mathrm{a}}$ Marie Standl $^{\mathrm{a}}$ Sibylle Koletzko ${ }^{\mathrm{b}}$ \\ Carl-Peter Bauer ${ }^{c} \quad$ Andrea von Berg $^{d}$ Dietrich Berdel ${ }^{d} \quad$ Ursula Krämer $^{\mathrm{e}}$ \\ Beate Schaaf $^{d}$ Irina Lehmann ${ }^{f}$ Olf Herbarth ${ }^{g}$ Joachim Heinrich ${ }^{a}$ \\ for the GINI-plus and LISA-plus Study Groups \\ a Helmholtz Zentrum München, German Research Center for Environmental Health, Institute of Epidemiology I, \\ Neuherberg, b Dr. von Hauner Children's Hospital, Ludwig Maximilians University of Munich, \\ 'Department of Pediatrics, Technical University of Munich, Munich, ${ }^{d}$ Department of Pediatrics, Marien-Hospital \\ Wesel, Wesel, e Institut für Umweltmedizinische Forschung, University of Düsseldorf, Düsseldorf, \\ ${ }^{\mathrm{f} C e n t e r ~ f o r ~ E n v i r o n m e n t a l ~ R e s e a r c h, ~ a n d ~}{ }^{\mathrm{g}}$ Environmental Medicine and Hygiene, Faculty of Medicine, \\ University Leipzig, Leipzig, Germany
}

\section{Key Words}

Behavior problems · Diet - Epidemiology · Depression • Attention deficit hyperactivity disorder -

Psychopathology • Mental health · Child psychiatry • Nutrition $\cdot$ Hyperactivity $\cdot$ Inattention

\begin{abstract}
Background/Aims: To assess the association between food intake and diet quality and behavioral problems at the 10year follow-up of the two population-based birth cohorts of the studies German Infant Nutritional Intervention and 'Influences of lifestyle-related factors on the immune system and the development of allergies in childhood'. Methods: Cross-sectional data on food intake over the past year were collected by a parent-reported food frequency questionnaire. Diet quality was based on reference values of food amounts of the optimized mixed diet. Behavioral problems were assessed by a parent-reported Strengths and Difficulties Questionnaire. Relationships between food category intake, diet quality and behavior problems were examined us-
\end{abstract}

ing multivariable regression modeling adjusted for gender, sociodemographic characteristics, body mass index, physical exercise, television viewing/PC use and total energy intake. A total of 3,361 children with complete data were analyzed. Results: Children with increased intake of confectionery had increased odds of having emotional symptoms [adjusted odds ratio $\left(\mathrm{OR}_{\mathrm{adj}}\right) 1.19,95 \%$ confidence interval $(\mathrm{Cl})$ 1.08-1.32] compared to children with low intake. A higher diet quality score was associated with lower likelihood of emotional symptoms $\left(\mathrm{OR}_{\mathrm{adj}} 0.89,95 \% \mathrm{Cl} 0.80-0.98\right)$. The unadjusted significant relationship between diet quality and hyperactivity/inattention was attenuated by adjusting for several confounders to an $\mathrm{OR}_{\text {adj }}$ of 0.92 (95\% Cl 0.82-1.03). Conclusions: Increased consumption of high-sugar products and lower diet quality are associated with a higher likelihood of emotional symptoms in children.

Copyright $\odot 2012$ S. Karger AG, Basel

For full details of the GINI-plus and LISA-plus Study Groups, see the Appendix.

\section{KARGER \\ Fax +41613061234 \\ E-Mail karger@karger.ch}

www.karger.com
(C) 2012 S. Karger AG, Basel

$0250-6807 / 12 / 0604-0247 \$ 38.00 / 0$

Accessible online at:

www.karger.com/anm
Gabriele Kohlboeck

Helmholtz Zentrum München, Institut für Epidemiologie

Ingolstädter Landstrasse 1

DE-85764 Neuherberg (Germany)

Tel. +49893187 4578, E-Mail gabriele.kohlboeck@helmholtz-muenchen.de 


\section{Introduction}

Diet is one of the most obvious yet underrecognized factors in mental health [1]. Evidence from a small but growing set of observational and interventional studies indicates that modifications of the lifestyle factor 'diet' can promote mental health $[2,3]$. Potential effects of nutritional factors on behavior are of special importance in childhood and adolescence, and attention deficit hyperactivity disorder (ADHD) has attracted much attention regarding the presence of certain elements in the diet $[4,5]$. Only one previous study exists that investigated the role of dietary patterns in mental health in a population-based sample of children [6]. In that study, an increased 'junk food' consumption at age 4 and $1 / 2$ years was associated with increased hyperactivity at age 7 , but there was no evidence for emotional problems. Contrary to this finding, three recent Australian studies reported relationships between diet quality and depressive symptoms in adolescents $[3,7,8]$. In these studies, depression was associated with a lower healthy diet score $[3,8]$ and with a Western dietary pattern consisting of increased intakes of takeaway foods, confectionery and red meat [7]. Similar relationships with depression were also reported from several adult studies investigating healthy dietary patterns $[9,10]$, unhealthy dietary patterns [11-13] and diet quality [14].

So far, no study has investigated the role of food intake and diet quality in mental health in a population-based sample of children using an overall diet approach. In this cross-sectional study, we aimed to investigate the relationships between a child's usual food intake over the past year from a parent-reported food frequency questionnaire (FFQ) and diet quality and behavioral problems from a parent-reported behavioral screening questionnaire. We hypothesized that higher consumption of highfat and high-sugar food categories would be associated with a higher likelihood of behavioral problems. We further hypothesized that diet quality, based on a quantitative German optimized mixed diet (OMD) concept for children and adolescents [15], would be associated with a lower likelihood of behavior problems.

\section{Material and Methods}

\section{Study Population}

Data from two ongoing German birth cohort studies were combined for the present analysis. The German Infant Nutritional Intervention (GINI-plus) study is a prospective birth cohort study to investigate the influence of nutritional intervention during infancy as well as air pollution and genetics on allergy devel- opment $[16,17]$. A total of 5,991 healthy full-term newborns were recruited in obstetric clinics in two regions of Germany (urban Munich and rural Wesel) between September 1995 and June 1998. Of the original study population, 3,317 children (55.4\%) participated in the 10 -year follow-up. Loss to follow-up was associated with a lower level of parental education, a negative history of parental atopy, the absence of atopic diseases in the child during the first 2 years of life and residency in Wesel.

The 'Influences of lifestyle-related factors on the immune system and the development of allergies in childhood' (LISA-plus) study $[18,19]$ is a population-based birth cohort of 3,097 newborns recruited between November 1997 and January 1999 in the four German cities Munich, Leipzig, Wesel and Bad Honnef. Of the original study population, 1,761 children (56.9\%) participated in the 10-year follow-up. Loss to follow-up was strongly associated with a lower level of parental education and residency in Wesel or Leipzig.

Of the 3,435 subjects from GINI-plus $(\mathrm{n}=2,190)$ and LISAplus $(\mathrm{n}=1,245)$ with FFQ data, complete data were available for 3,361 participants $(1,712$ males, $51 \% ; 1,649$ females, $49 \%)$ at the 10 -year follow-up. These data constituted the sample which is presented in this paper.

For both studies, approval by the local ethics committees (Bavarian General Medical Council, University of Leipzig, Medical Council of North Rhine-Westphalia) and written consent from the participants' families were obtained.

\section{Measurement of Behavioral Problems}

The parent-reported Strengths and Difficulties Questionnaire (SDQ) [20] is a brief behavioral screening questionnaire for children which comprises 25 items on psychological attributes divided into 5 subscales with scores that range from 0 to 10 , where higher scores denote more problems. For the present analysis, the three subscales emotional symptoms, conduct problems and hyperactivity/inattention were used to cover the most important current domains of child psychopathology. SDQ subscales were categorized into three groups according to the norms of the German SDQ version [21], indicating a 'normal', 'borderline' or 'abnormal' degree of symptoms. Cutoff values for German norms resulted in a prevalence rate of $25 \%$ for abnormal/borderline conduct problems and were adjusted for our sample using a cutoff value of 3.75 .

\section{Food Intake}

The 82-item FFQ administered to the parents was designed to measure the children's usual food intake over the past year. The effectiveness of this questionnaire to collect dietary data to draw inferences concerning the effect of nutrient intake on disease states has been confirmed [22]. To estimate how often foods were consumed by their child on average over the previous year, parents were asked to choose one of nine frequency categories, ranging from 'never' to 'four times a day or more'. In addition, common portion sizes were given for each food to enable an estimation of quantities. The consumption frequencies and portion size estimates were converted into average consumption in grams per day. The 82 FFQ food items were collapsed into 11 food categories using the Codex General Standard for Food Additives food category system of the Codex Alimentarius Commission of the Food and Agriculture Organization of the United Nations/World Health Organization [23]. The Codex General Standard for Food 
Additives food category system is hierarchical and includes a description of the foods covered by each food category. Food category data are presented as means (SD) and 25th and 75th percentiles stratified by gender.

\section{Diet Quality}

From the average food consumption in grams per day (from the FFQ), a diet quality score was constructed, whereby a point was allotted for each agreement with the food amounts of the 11 components of the OMD, a quantitative preventive dietary concept for children and adolescents which takes German meal patterns into account. The OMD was developed by the German Research Institute of Child Nutrition and applied Food and Agriculture Organization of the United Nations/World Health Organization standards for Food Based Dietary Guidelines [15, 24]. Compared to the existing German high-fat, low-plant food diet, the OMD is lower in fat and saturated fatty acids and contains ample amounts of plant foods. In the OMD, 'recommended' foods are distinguished from 'tolerated' food by their nutrient densities (for more details, see table 3). Correspondence of FFQ food consumption with the reference value was coded as 1 ; otherwise it was coded as 0 . The OMD diet quality score is the sum of these dichotomous variables.

\section{Covariates}

We adjusted for a variety of factors that could potentially confound the relationship between food intake and mental health. These covariates were selected based on the references of the previous studies $[6-8,25]$ and were determined a priori as follows: gender, study center (Munich, Wesel, Leipzig, Bad Honnef), parental education, parental income, single-parent family $(0=$ no, $1=$ yes), body mass index (BMI), physical exercise, television viewing/PC use and total energy intake. Parental education was determined on the basis of the German educational system and by the highest grade completed by either the mother or the father. Thus, children were assigned to the group of low (less than tenth grade), medium (tenth grade) or high (more than tenth grade) parental education. Net household income per month was reported in the 10 -year questionnaire using a 9-point scale ranging from $<$ EUR 500 to $>$ EUR 3,500. The calculation of equivalent income was carried out according to the Organization for Economic Cooperation and Development guidelines [26, 27] and grouped into low, medium and high household income. The BMI (weight in kilograms/ height in meters squared) was calculated from weight and height measured by a physician during the clinical examination. The children were asked how many hours they exercised outside of school per week, where physical activity was defined as activity causing breathlessness or sweating. These data were grouped based on distribution within the study population as high $(>75$ th percentile), medium (25th-75th percentile) and low physical activity ( $<25$ th percentile). Children were also asked about their TV or video viewing and computer (PC) use, including video games, and these data were grouped as low (less than $1 \mathrm{~h}$ per day), medium (1-2 h per day) and high ( $3 \mathrm{~h}$ or more per day). We adjusted for total energy intake (in megajoules) to ensure that observed associations were independent of the children's total energy intake [7].

\section{Data Analysis}

The Kolmogorov-Smirnov test and normal probability plots were used to test for normal distribution of food items. Student's $t$ test, the Wilcoxon two-sample test and $\chi^{2}$ tests were used to compare several variables between groups; means and SDs were used to describe continuous variables. A 0.05 level of significance was considered. Logistic regression was considered the best option to evaluate the effect of each food category separately on the dichotomously coded behavior problems (SDQ subscale categories: $0=$ normal, 1 = borderline or abnormal behavior difficulties) because the dependent variable demonstrated a skewed distribution, rendering linear regression models inappropriate. For logistic regressions, the individual intake of the 11 food categories derived from the FFQ was divided by its respective gender-specific interquartile range (IQR; the difference between the 25 th and 75 th percentile of the total sample). The predictors are expressed as IQR increase. The advantage of the IQR as a scaling factor is that it will always reflect values of the predictor that are relatively well-represented in the sample [28]. This method allows the interpretation of predictors as comparing a 'typical' person in the middle of the upper half of the predictor distribution (i.e. 75th percentile) with a 'typical' person in the middle of the lower half of the predictor distribution (25th percentile). Multiple testing was performed in order to reduce the type 1 error and to overcome the problem of false positive association. The overall number of tests was 33; by applying the Bonferroni correction, a corrected two-sided alpha level of $\mathrm{p}=0.0015$ was applied. To investigate whether or not diet quality is associated with behavioral problems, logistic regression analysis with diet quality score as the independent variable and dichotomously coded behavior problems as the dependent variable was used. Results are presented as odds ratios (ORs) with corresponding 95\% confidence intervals (CIs; without Bonferroni correction). All computations were performed using the statistical software package SAS for Windows, version 9.2 (SAS Institute, Cary, N.C., USA).

\section{Results}

Subjects were assessed about 10 years after the baseline survey at birth. At completion of the FFQ, the children were about 11 years old (mean age 11.15 years, SD 0.5, range 9.9-12.7). Characteristics of the GINI-plus/LISAplus study samples and the prevalence of behavior problems are shown in table 1 . There was a weak but significant difference in the distribution of parental education between boys and girls $(\mathrm{p}=0.05)$, with a higher proportion of high parental education for girls. There were no genderrelated differences with regard to parental income, singleparent families or BMI. Distribution of physical activity was significantly different $(\mathrm{p}<0.001)$, indicating a higher proportion of boys with high activity. Similarly, the distribution of daily TV or video/computer game use showed a higher proportion of boys watching TV or using the PC more often compared to girls $(\mathrm{p}<0.0001)$. Daily energy intake was significantly higher in boys (mean 9.3 MJ, SD 3.0) than girls (mean 7.8 MJ, SD 2.3; p <0.0001). The prevalence of borderline/abnormal behavior problems in the 
Table 1. Characteristics of the GINI-plus and LISA-plus study sample $(n=3,361)$ at the 10 -year follow-up

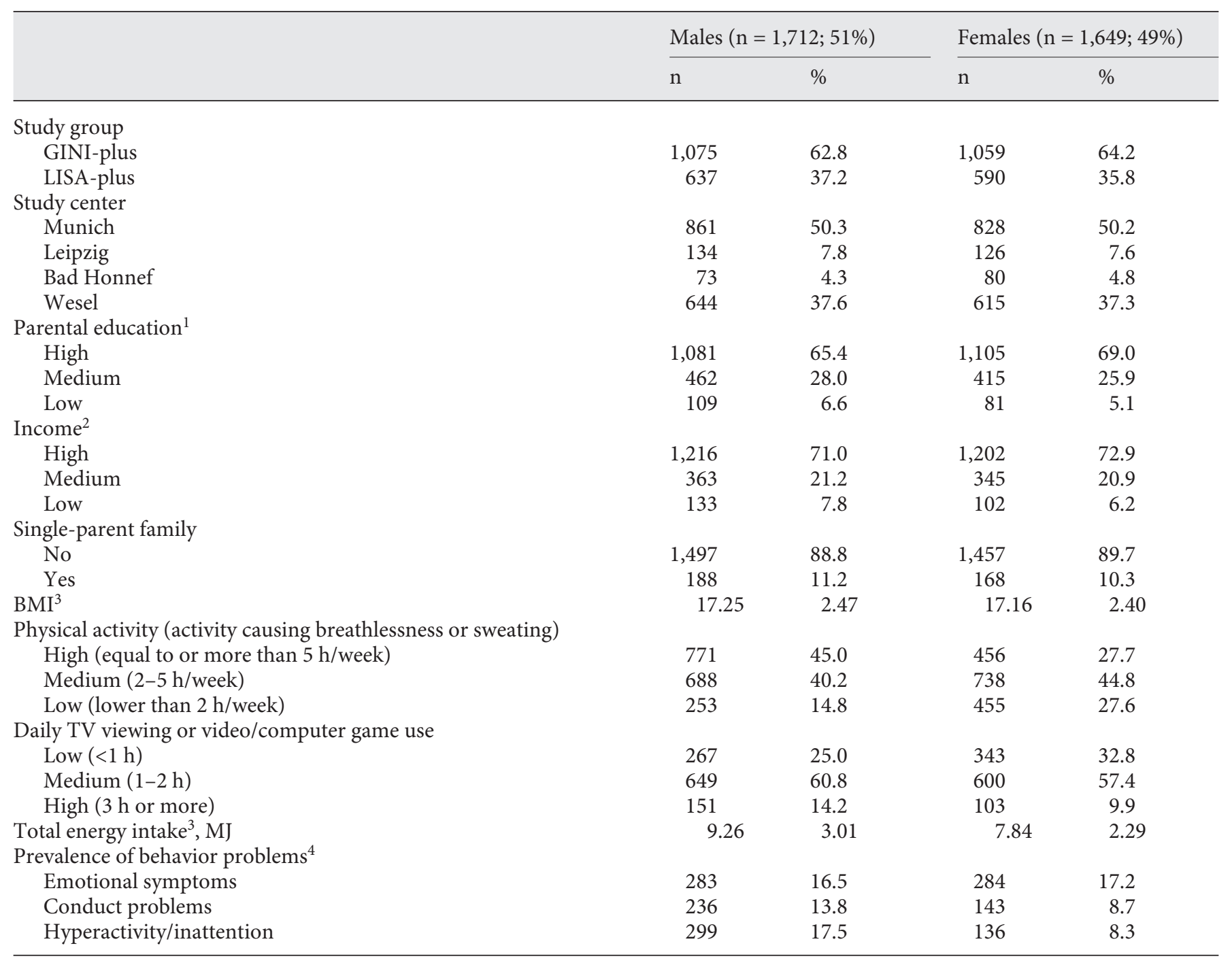

${ }^{1}$ Educational status based on the highest completed grade in school and vocational training of either parent: high $=$ more than tenth grade; medium = tenth grade; low = less than tenth grade. ${ }^{2}$ Parental income based on household equivalent income. ${ }^{3}$ Results shown as means and SDs. ${ }^{4}$ Emotional symptoms: score $=4$, borderline, score $>4$, abnormal; conduct problems: score $=3.75$, borderline, score $>3.75$, abnormal; hyperactivity/inattention: score $=6$, borderline, score $>6$, abnormal.

total sample was about $17 \%$ for emotional symptoms, $12 \%$ for conduct problems and $13 \%$ for hyperactivity/inattention. Boys showed significantly higher borderline/abnormal conduct problems $(\mathrm{p}<0.001)$ and hyperactivity/inattention $(\mathrm{p}<0.001)$ than girls.

There were significant differences in the distribution of consumption of all food categories between boys and girls (table 2). With the exception of fruits and vegetables, food consumption across the food categories was higher for boys than girls.
The distribution of correspondence of FFQ food intake with recommended intake for each component of the OMD diet is presented in table 3. The overall mean diet quality score (data not shown) based on the dichotomous value of correspondence with each of the $11 \mathrm{OMD}$ components $(1=$ yes, $0=$ no) was 2.80 (SD 1.22; minimum 0 , maximum 8$)$. The mean diet quality scores were similar for boys (mean 2.83, SD 1.22) and girls (mean 2.76, SD $1.22 ; \mathrm{p}=0.08$ ). As shown in table 3 , agreement with OMD reference values for plant foods was low. Only $1-2 \%$ of 
Table 2. Distribution of food intake data in 11 food categories in the study population by gender

\begin{tabular}{|c|c|c|c|c|c|c|c|c|c|c|}
\hline & & \multicolumn{4}{|c|}{ males $(\mathrm{n}=1,712)$} & \multicolumn{4}{|c|}{ females $(\mathrm{n}=1,649)$} & $\mathrm{p}$ value \\
\hline 1 & Dairy and dairy products (milk, cheese, yoghurt) & 477 & 403 & 226 & 609 & 360 & 296 & 167 & 449 & $<0.0001$ \\
\hline 2 & Fats and oils (butter, vegetable oils, margarine) & 18 & 13 & 9 & 23 & 16 & 11 & 8 & 21 & $<0.0001$ \\
\hline 5 & Cereals and cereal products (rice, pasta, breakfast cereals) & 95 & 59 & 55 & 119 & 82 & 49 & 50 & 74 & $<0.0001$ \\
\hline 6 & Bakery wares (bread, rolls, cakes, cookies) & 157 & 69 & 109 & 192 & 141 & 59 & 100 & 174 & $<0.0001$ \\
\hline 7 & Meat and meat products (fresh and processed meat, sausages) & 126 & 88 & 73 & 153 & 99 & 66 & 58 & 122 & $<0.0001$ \\
\hline 8 & Fish and fish products & 21 & 16 & 10 & 28 & 18 & 18 & 7 & 24 & $<0.0001$ \\
\hline 9 & Eggs and egg products & 26 & 26 & 11 & 30 & 22 & 20 & 10 & 26 & $<0.0001$ \\
\hline 10 & $\begin{array}{l}\text { Beverages (soft and energy drinks, fruit juices/nectars, water-based } \\
\text { flavored drinks, tea) }\end{array}$ & 673 & 477 & 288 & 960 & 582 & 413 & 243 & 857 & $<0.0001$ \\
\hline
\end{tabular}

Food categories were defined according to the Codex General Standard for Food Additives food category system [23]. p values were calculated using the Wilcoxon two-sample test. P25 = 25th percentile; P75 = 75th percentile.

girls and boys met the criterion for potatoes/pasta/rice and about $4-7 \%$ for vegetables and fruit. The criterion for moderate animal food was met by between 15 and $24 \%$ of our sample. The criterion for tolerated food was met by significantly more girls $(32.4 \%)$ than boys $(25 \%$; p < 0.0001).

\section{Association between Intake of Food Categories and Behavioral Problems}

The associations of the gender-specified interquartile increase (25-75\%) of the 11 food categories with abnormal or borderline behavioral problems were investigated by logistic regression models. Results of unadjusted analyses showed statistically significant, albeit modest associations between several food categories and behavioral problems. Bonferroni-corrected results showed that intake of confectionery was significantly associated with emotional symptoms. Children with increased intake have a $12 \%$ increased odds of having emotional symptoms compared to children with low intake (unadjusted OR 1.12, 95\% CI 1.05-1.19). Adjustment for confounders (gender, study center, total energy intake, parental socioeconomic background, BMI, physical activity, TV viewing or video/computer game use) showed that children with increased consumption of confectionery had an approximately $20 \%$ higher odds of having emotional symptoms [adjusted OR $\left(\mathrm{OR}_{\mathrm{adj}}\right) 1.19,95 \%$ CI 1.08-1.32; fig. 1]. However, in these analyses none of
Table 3. Distribution of adherence to OMD intake in our study sample

\begin{tabular}{ll}
\hline Components & $\begin{array}{l}\text { Total group } \\
(\mathrm{n}=3,361)\end{array}$ \\
\cline { 2 - 2 } & $\mathrm{n} \%$ \\
\hline
\end{tabular}

Ample beverages and plant foods

1 Beverages $(\geq 1,000 \mathrm{~g} /$ day $)$

$1,663 \quad 49.5$

2 Vegetables ( $\geq 250$ g/day) $\quad 227 \quad 6.8$

3 Fruit $(\geq 250 \mathrm{~g} /$ day $) \quad 154 \quad 4.6$

4 Potatoes/pasta/rice ( $\geq 270$ g/day $) \quad 52 \quad 1.6$

5 Bread/cereals ( $\geq 250$ g/day) $\quad 482 \quad 14.3$

Moderate animal foods

$6 \mathrm{Milk} / \mathrm{milk}$ products (336-504 g/day) $\quad 676 \quad 20.1$

7 Meat/sausages (48-72 g/day) $\quad 603 \quad 17.9$

8 Eggs (2-3 per week) $\quad 561 \quad 16.7$

9 Fish (10-16 g/day) $806 \quad 24.0$

Sparing high-fat, high-sugar foods

10 Oil/fats (35 g/day) $\quad 838 \quad 24.9$

11 Tolerated food groups (max. $220 \mathrm{kcal} /$ day) $\quad 955 \quad 28.4$

Tolerated FFQ food items were ice cream, cookies, biscuits, cakes, croissants, pastries, pies, fruit gums, chocolate, chocolate bars, nougat crème, pudding, chips (crisps), soft drinks, cola and energy drinks. 
Fig. 1. Intake of food categories and behavioral problems. ORs were adjusted for gender, study center, sociodemographic characteristics, BMI, physical exercise, television viewing/PC use and total energy intake (ORs not Bonferroni corrected).

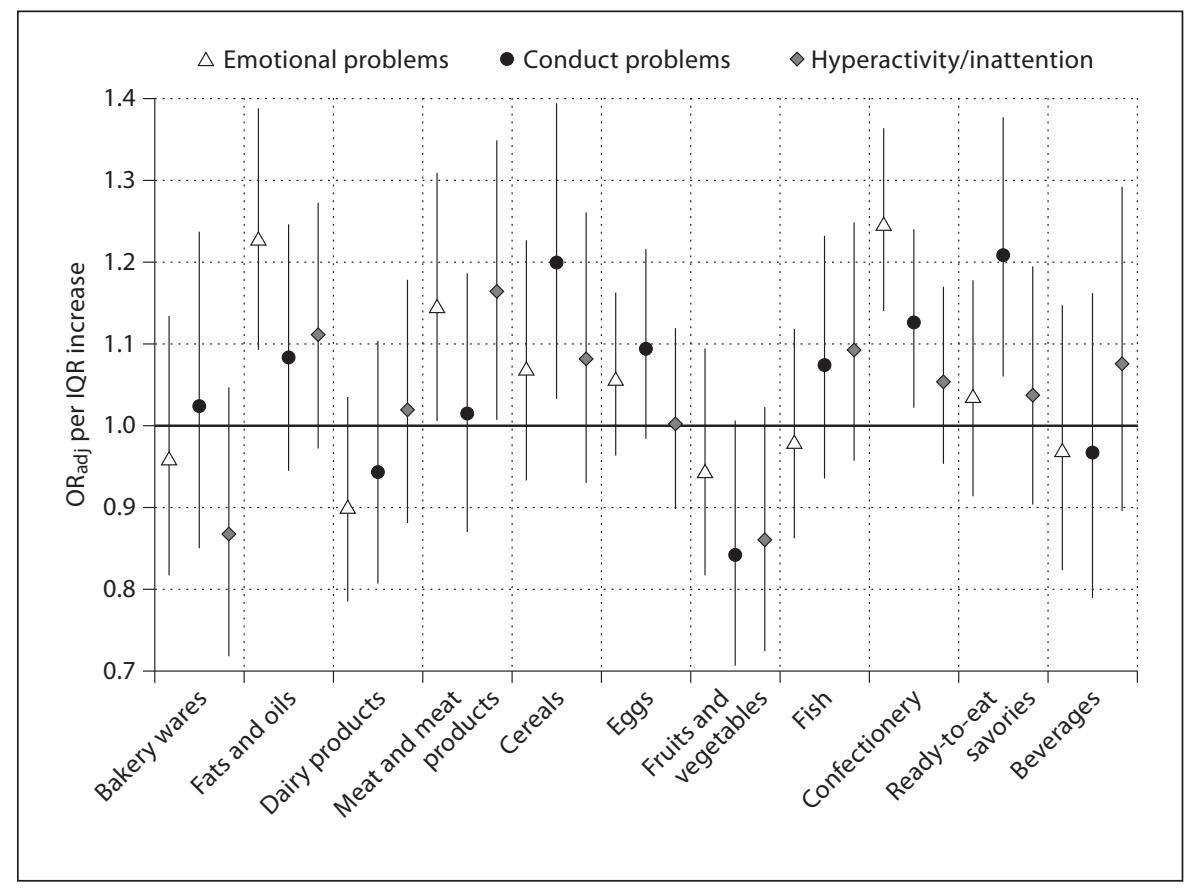

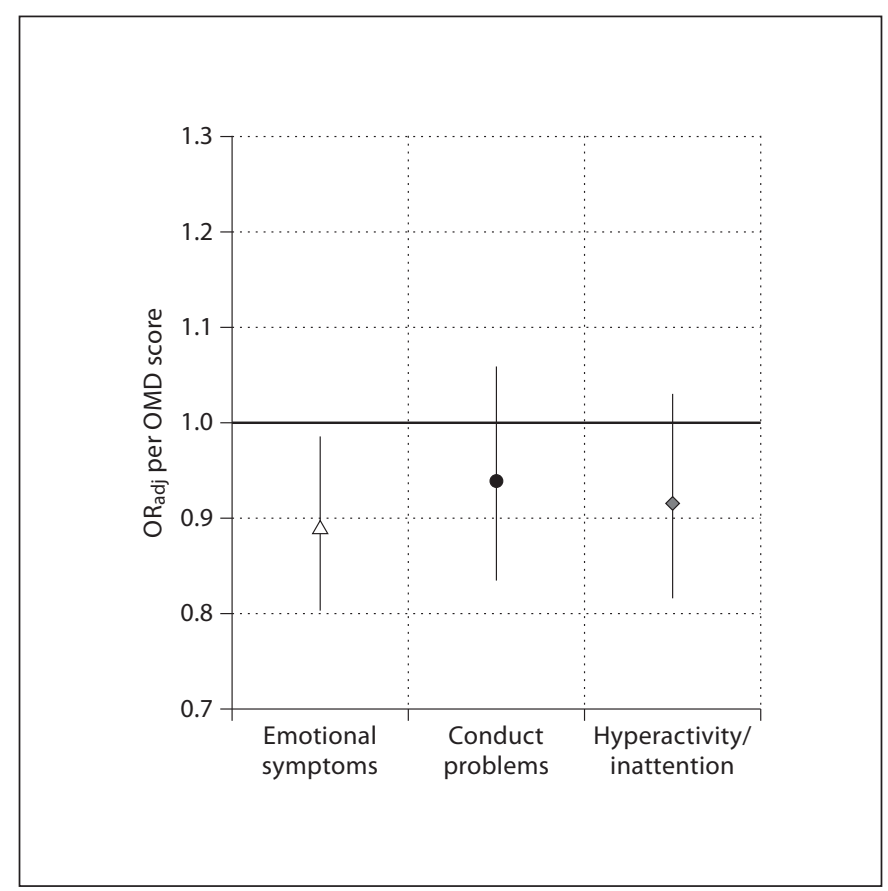

Fig. 2. Association between OMD quality and behavioral problems. ORs were adjusted for gender, study center, sociodemographic characteristics, BMI, physical exercise, television viewing/PC use and total energy intake. the other food categories showed significant associations with behavioral problems (with Bonferroni correction).

\section{Association between OMD Diet Quality and \\ Behavioral Problems}

Results of unadjusted analyses for the diet quality score based on correspondence with the 11 OMD components showed that diet quality was significantly associated with emotional symptoms $(\mathrm{p}<0.02)$ and hyperactivity/inattention $(\mathrm{p}<0.03)$, but there was no significant relationship with conduct problems (fig. 2). The ORs indicate that each unit increment (1.0) in diet quality reduces the odds of emotional problems by a factor of 0.91 (95\% CI $0.85-0.98)$ and of hyperactivity/inattention by 0.91 (95\% CI 0.83-0.99). When controlled for confounders (gender, study center, parental education, household income, single-parent family, BMI, physical activity, TV viewing or video/computer game use, total energy intake), the association between diet quality and emotional problems remained significant $\left(\mathrm{OR}_{\mathrm{adj}} 0.89,95 \%\right.$ CI $0.80-$ $0.98)$. There were no significant associations between diet quality and conduct problems $\left(\mathrm{OR}_{\mathrm{adj}} 0.94,95 \% \mathrm{CI} 0.83-\right.$ 1.06). However, the relationship between diet quality and hyperactivity/inattention was attenuated by parental socioeconomic background and BMI ( $\mathrm{OR}_{\text {adj }} 0.92,95 \% \mathrm{CI}$ $0.82-1.03)$. 


\section{Discussion}

Our results show that increased intake of high-sugar food ('confectionery') was associated with a $20 \%$ higher likelihood of emotional symptoms compared to low intake. The OMD diet quality score reflecting a diet low in fat but ample amounts of plant foods indicates that higher diet quality is associated with lower likelihood of emotional symptoms and hyperactivity/inattention. However, the relationship between diet quality and hyperactivity/inattention was attenuated when controlled for parental education, single-parent family and BMI.

Our results on the relationship between confectionery consumption and emotional symptoms are not in line with a previous prospective study which found no evidence for an association between sugar intake or junk food at the age of 4 and $1 / 2$ years and emotional problems at age 7 [6]. The differences between these studies might be explained by age effects. At a younger age, confectionery intake may be restricted by greater parental control than in older children. The association may become more pronounced as children age. This explanation is supported by findings in adolescents indicating that the highest quartile for confectionery intake was associated with higher depression scores [7]. Our result also corresponds with a cross-sectional study of 1,018 adults that found higher depression scores to be related to greater chocolate consumption [13].

Although diet quality in our study was measured differently from previous research, our finding of an inverse relationship between OMD diet quality and emotional problems is similar to previous findings in adolescents [3, $7,8]$ and adults [14]. An Australian study reported a lower healthy diet score, based on breakfast before school and daily servings of low-fat dairy food, fruits and vegetables, to be associated with increased odds of self-reported symptomatic depression at age 10-14 [8]. A similar relationship was reported from another cross-sectional study of Australian adolescents using a factor analysisgenerated Western dietary pattern [7]. In addition, a recent prospective study of Australian adolescents aged 1118 years found a higher healthy diet score (based on eating breakfast at home, eating lunch brought from home, healthy snacks, consuming fruits and vegetables and generally avoiding fried foods, chocolate, sweets, etc.) to be predictive of lower depressive symptoms at follow-up 2 years later [3]. With regard to the prospective results, the authors suggest that the reverse causality hypothesis, i.e. that poorer eating habits are a consequence of mental health problems, was not supported by their data [3]. Sev-

Food Intake and Child Behavior Problems eral adult studies also showed evidence for a relationship between depression and diet quality by applying a Western dietary pattern [11] or a healthy eating index from Dietary Guidelines for Americans [14].

However, children with an OMD did not show any substantial behavioral problems or, expressed the other way around, those children with no behavioral problems obviously adhered to an OMD.

Jacka and Berk [29] suggest that the typical dietary patterns of industrialized societies (a diet high in saturated fats and refined sugar, with energy-dense, nutrientpoor foods and a corresponding reduction in fruit, vegetables and fiber) may have an impact on the prevalence rates of depression in the community. Our results also point in this direction for schoolchildren. We found that adherence to a diet lower in fat and saturated fatty acids and with higher amounts of plant foods that met nutrient density recommendations [30] was associated with reduced odds for emotional symptoms.

The proinflammation theory of depression offers an explanation for our results. Diets high in fat and sugar promote insulin resistance, which in turn contributes to systematic inflammation [31]. The proinflammatory cytokines are thought to directly mediate many of the behavioral neuroendocrine and neurochemical changes seen in depressive illness [32].

Compared to a previous study on adolescents that found higher consumption of leafy green vegetables to be associated with lower conduct problems and higher intake of confectionery, takeaway foods, red meat and potato crisps to be associated with more conduct problems [7], we found only weak support for an association with conduct problems. An explanation for the inconsistent results may be the use of different methodological approaches to assess dietary patterns. Since our results agree with a previous study on children at age 7 that found no evidence for an association between 'junk food' intake and conduct problems [6], we suggest age-related effects as another explanation.

With regard to hyperactivity/inattention, we found only weak associations with decreased intake of 'vegetables and fruit' and increased 'meat and meat products'. Results for 'meat and meat products' agree with findings that foods high in fat are associated with hyperactivity/ inattention [6]. Our finding that an OMD is associated with reduced hyperactivity/inattention at age $11 \mathrm{did}$ not hold when the factors parental education, BMI and single-parent family were controlled for. Similarly, Wiles et al. [6] reported the 'junk food' factor to be weak when socioeconomic variables were controlled for. A possible 
explanation is that parental educational style may be a factor in the relationship between high-fat/high-sugar food and hyperactivity $[5,6]$, or permanent nutritional imbalances [6].

Previous research on ADHD focused mainly on the potential effects of allergenic foods and on diet interventions that restrict food additives, preservatives and sugar [33], and evidence from clinical trials suggests an effect of artificial food colorings and preservatives on hyperactivity [34, 35]. Schnoll et al. [5] suggest that there might not be a specific food or substance that will precipitate hyperactivity, but individual allergens and sensitivities. A recent randomized clinical trial found that an individual restricted elimination diet had a significant beneficial effect on ADHD symptoms in children [36]. Even though the OMD diet is different from the restricted elimination diet approach, which consists of the few-foods diet (i.e. rice, meat, vegetables, pears and water), our results support evidence that suggests that dietary intervention should be considered in children with ADHD [36].

\section{Strengths and Limitations}

The strength of our study, apart from its large number of population-based subjects, is that it offers the possibility to investigate the parent-reported overall view of the diet of their child and adherence with reference values of an OMD in relation to behavior problems. Using the OMD approach for diet quality had the advantage that exact quantification of foods was given to ascertain nutrient adequacy [15]. Our results are based on data from two independent population-based birth cohorts. Stratified analysis for both studies showed similar results. Aiming for a homogeneous study population, we included only healthy newborns of German nationality; subjects with low birth weight or born preterm were excluded.

However, because of the cross-sectional nature of our data, the direction of the relationship between behavior problems and food remains unclear, i.e. whether unhealthy food affects behavior problems or behavior problems affect choices of unhealthy food. An alternative explanation for an association between depression and unhealthy food patterns is that subjects with depression consume fatty foods and sweet foods in an attempt to improve mood [37]. It is also likely that the relationships between negative emotions and food consumption are bidirectional, i.e. the food consumed affects mood and mood affects food choices [38]. Another limitation of our study was the use of questionnaires which provide no exact diagnoses of mental disorders or exact data on food intake. However, we used the standardized and well-val- idated brief SDQ behavioral screening, which has been especially developed for use in epidemiological studies. The FFQ is a preferable method for large studies and collects portion size information as standardized portions. Due to our study design, we had to rely solely on parental report of behavioral problems and diet intake, and reporting biases limit generalization of our results to parent-rated data. Aspects of parental psychopathology, family management and parental style that are likely to have an impact on both diet and mental health in childhood were not included as confounders but might have an effect on our results.

\section{Conclusions}

Our finding that a higher diet quality is associated with reduced odds of behavioral problems supports the notion that good regular dietary habits are the best way to ensure optimal mental and behavioral performance [4]. Future studies may investigate whether reducing foods high in sugar and fat would reduce mental disorders in clinical populations and determine in a longitudinal design how dietary interventions could decrease behavioral problems. It would be a pivotal change for psychiatry if specific dietary patterns are definitively demonstrated to prevent or diminish the prevalence or severity of psychiatric disorders [39]. The healthcare system might give greater attention to nutritional factors in mental health, since nutrition interventions can be inexpensive, safe, easy to administer and generally acceptable to patients [40]. Finally, a potential intervention by reducing foods high in sugar content and highly processed fatty food and increasing fruit consumption is known to be a prudent diet and highly recommended for many other reasons than to prevent behavior problems.

\section{Appendix}

The GINI-plus Study Group comprises the following centers: Helmholtz Zentrum München, German Research Center for Environmental Health, Institute of Epidemiology, Munich (J. Heinrich, H.E. Wichmann, S. Sausenthaler, A. Zutavern, C.M. Chen, M. Schnappinger, P. Rzehak); Department of Pediatrics, MarienHospital Wesel, Wesel (D. Berdel, A. von Berg, C. Beckmann, I. Gross); Department of Pediatrics, Ludwig Maximilians University, Munich (S. Koletzko, D. Reinhardt, S. Krauss-Etschmann); Department of Pediatrics, Technical University, Munich (C.P. Bauer, I. Brockow, A. Grübl, U. Hoffmann); IUF - Institut für Umweltmedizinische Forschung, Heinrich Heine University, Düsseldorf (U. Krämer, E. Link, C. Cramer), and the Center for 
Allergy and Environment, Technical University, Munich, Germany (H. Behrendt).

The LISA-plus Study Group comprises the following centers: Helmholtz Zentrum München, German Research Center for Environmental Health, Institute of Epidemiology, Munich (J. Heinrich, H.E. Wichmann, S. Sausenthaler, C.M. Chen, M. Schnappinger); Department of Pediatrics, Municipal Hospital 'St. Georg', Leipzig (M. Borte, U. Diez); Department of Pediatrics, MarienHospital Wesel, Wesel (A. von Berg, C. Beckmann, I. Gross); Pediatric Practice, Bad Honnef (B. Schaaf); Department of Environmental Immunology/Core Facility Studies, Helmholtz Center for Environmental Research, Leipzig (I. Lehmann, M. Bauer, C. Gräbsch, S. Röder, M. Schilde); Institute of Hygiene and Environmental Medicine, University of Leipzig, Leipzig (O. Herbarth, C. Dick, J. Magnus); IUF - Institut für Umweltmedizinische Forschung, Düsseldorf (U. Krämer, E. Link, C. Cramer); Department of Pediatrics, Technical University Munich, Munich (C.P. Bauer, U. Hoffmann), and the Center for Allergy and Environment, Technical University, Munich, Germany (H. Behrendt, J. Grosch, F. Martin).

\section{Acknowledgements}

This research was funded by grants 01 EG 9732 and 01 EG 9705/2 (LISA Study) and 01 EE 9401-4 (GINI Study) from the Federal Ministry for Education, Science, Research and Technology, grant FKZ 20462296 from the Federal Ministry of Environment and the Ludwig Maximilians University's innovative research priority project MC-Health (GINI Study).

\section{Disclosure Statement}

No conflict of interest declared.

\section{References}

1 Cornah D: Feeding Minds: The Impact of Food on Mental Health. London, Mental Health Foundation, 2005.

2 O'Connell ME, Boat T, Warner KE: Preventing Mental, Emotional, and Behavioral Disorders Among Young People: Progress and Possibilities. Washington, National Academy of Sciences, 2009.

3 Jacka FN, Kremer PJ, Berk M, et al: A prospective study of diet quality and mental health in adolescents. PLoS One 2011; 6:e24805.

4 Bellisle F: Effects of diet on behaviour and cognition in children. Br J Nutr 2004;92(suppl 2):S227-S232.

5 Schnoll R, Burshteyn D, Cea-Aravena J: Nutrition in the treatment of attention-deficit hyperactivity disorder: a neglected but important aspect. Appl Psychophysiol Biofeedback 2003;28:63-75.

6 Wiles NJ, Northstone K, Emmett P, Lewis G: 'Junk food' diet and childhood behavioural problems: results from the ALSPAC cohort. Eur J Clin Nutr 2009;63:491-498.

7 Oddy WH, Robinson M, Ambrosini GL, et al: The association between dietary patterns and mental health in early adolescence. Prev Med 2009;49:39-44.

8 Jacka FN, Kremer PJ, Leslie ER, et al: Associations between diet quality and depressed mood in adolescents: results from the Australian Healthy Neighbourhoods Study. Aust NZ J Psychiatry 2010;44:435-442.

9 Jacka FN, Pasco JA, Mykletun A, et al: Association of Western and traditional diets with depression and anxiety in women. Am J Psychiatry 2010;167:305-311.
10 Nanri A, Kimura Y, Matsushita Y, et al: Dietary patterns and depressive symptoms among Japanese men and women. Eur J Clin Nutr 2010;64:832-839.

11 Jacka FN, Pasco JA, Mykletun A, et al: Diet quality in bipolar disorder in a populationbased sample of women. J Affect Disord 2011;129:332-337.

12 Akbaraly TN, Brunner EJ, Ferrie JE, Marmot MG, Kivimaki M, Singh-Manoux A: Dietary pattern and depressive symptoms in middle age. Br J Psychiatry 2009;195:408-413.

13 Rose N, Koperski S, Golomb BA: Mood food: chocolate and depressive symptoms in a cross-sectional analysis. Arch Intern Med 2010;170:699-703.

14 Kuczmarksi M, Cremer Sees A, Hotchkiss L, Cotugna N, Evans MK, Zonderman AB: Higher Healthy Eating Index-2005 scores associated with reduced symptoms of depression in an urban population: findings from the Healthy Aging in Neighborhoods of Diversity Across the Life Span (HANDLS) study. J Am Diet Assoc 2010;110:383-389.

15 Kersting M, Alexy U, Clausen K: Using the concept of Food Based Dietary Guidelines to Develop an Optimized Mixed Diet (OMD) for German children and adolescents. J Pediatr Gastroenterol Nutr 2005;40:301-308.

16 Filipiak B, Zutavern A, Koletzko S, et al: Solid food introduction in relation to eczema: results from a four-year prospective birth cohort study. J Pediatr 2007;151:352-358.

17 von Berg A, Koletzko S, Filipiak-Pittroff B, et al: Certain hydrolyzed formulas reduce the incidence of atopic dermatitis but not that of asthma: three-year results of the German Infant Nutritional Intervention Study. J Allergy Clin Immunol 2007;119:718-725.
18 Heinrich J, Bolte G, Holscher B, et al: Allergens and endotoxin on mothers' mattresses and total immunoglobulin E in cord blood of neonates. Eur Respir J 2002;20:617-623.

19 Schnabel E, Sausenthaler S, Schaaf B, et al: Prospective association between food sensitization and food allergy: results of the LISA birth cohort study. Clin Exp Allergy 2010;40: 450-457.

20 Goodman R: The Strengths and Difficulties Questionnaire: a research note. J Child Psychol Psychiatry 1997;38:581-586.

21 Woerner W, Becker A, Rothenberger A: Normative data and scale properties of the German parent SDQ. Eur Child Adolesc Psychiatry 2004;13(suppl 2):II3-II10.

22 Stiegler P, Sausenthaler S, Buyken AE, et al: A new FFQ designed to measure the intake of fatty acids and antioxidants in children. Public Health Nutr 2010;13:38-46.

23 Food and Agriculture Organization of the United Nations/World Health Organization: Codex General Standard for Food Additives food category system. 2011. http://www.codexalimentarius.net/gsfaonline/foods/index.html (accessed 10 November 2011).

24 Alexy U, Sichert-Hellert W, Kersting M, Lausen B, Schoch G: Development of scores to measure the effects of nutrition counselling on the overall diet: a pilot study in children and adolescents. Eur J Nutr 1999;38:196200.

25 Krabbendam L, Bakker E, Hornstra G, van Os J: Relationship between DHA status at birth and child problem behaviour at 7 years of age. Prostaglandins Leukot Essent Fatty Acids 2007;76:29-34. 
26 Hauser R: Adequacy and poverty among the retired. Ageing Working Papers AWP 3.2. Paris, Organisation for Economic Co-operation and Development, 1988.

27 Sausenthaler S, Kompauer I, Mielck A, et al: Impact of parental education and income inequality on children's food intake. Public Health Nutr 2007;10:24-33.

28 Babyak MA: Rescaling continuous predictors in regression models [http://stattips. blogspot.com/2009/08/rescaling-continuous-predictors-in.html]. In Statistical Tips from the Editors of Psychosomatic Medicine 2009. Retrieved May, 19, 2011.

29 Jacka FN, Berk M: Food for thought. Acta Neuropsychiatr 2007;19:321-323.

30 Deutsche Gesellschaft fuer Ernaehrung: Reference values of nutrient intakes. Frankfurt, Deutsche Gesellschaft fuer Ernaehrung, 2000.
31 Weisberg SP, McCann D, Desai M, Rosenbaum M, Leibel RL, Ferrante AW Jr: Obesity is associated with macrophage accumulation in adipose tissue. J Clin Invest 2003;112: 1796-1808.

32 Schiepers OJ, Wichers MC, Maes M: Cytokines and major depression. Prog Neuropsychopharmacol Biol Psychiatry 2005;29:201217.

33 Wolraich M: Attention deficit hyperactivity disorder. Prof Care Mother Child 1998;8:3537.

34 Bateman B, Warner JO, Hutchinson E, et al: The effects of a double blind, placebo controlled, artificial food colourings and benzoate preservative challenge on hyperactivity in a general population sample of preschool children. Arch Dis Child 2004;89:506-511.

35 Schab DW, Trinh NH: Do artificial food colors promote hyperactivity in children with hyperactive syndromes? A meta-analysis of double-blind placebo-controlled trials. J Dev Behav Pediatr 2004;25:423-434.
36 Pelsser LM, Frankena K, Toorman J, et al: Effects of a restricted elimination diet on the behaviour of children with attention-deficit hyperactivity disorder (INCA study): a randomised controlled trial. Lancet 2011;377: 494-503.

37 Christensen L: The effect of food intake on mood. Clin Nutr 2001;20:161-166.

38 Gibson EL: Emotional influences on food choice: sensory, physiological and psychological pathways. Physiol Behav 2006;89:5361.

39 Freeman MP: Nutrition and psychiatry. Am J Psychiatry 2010;167:244-247.

40 Bodnar LM, Wisner KL: Nutrition and depression: implications for improving mental health among childbearing-aged women. Biol Psychiatry 2005;58:679-685. 